\section{Artigo Original}

\section{Original Article}

\section{Efeitos de campo operacional de instrução sobre aptidão física, dano muscular e controle autonômico de alunos do NPOR do Exército Brasileiro \\ Effects of Operational Field of Instruction on Physical Fitness, Muscle Damage Markers and Autonomic Control in NPOR Students of Brazilian Army}

Léo Dutra Cabistany ${ }^{1 \S}$ MS; Victor Silveira Coswig ${ }^{1,2}$ MS; Marcelo Vaz ${ }^{1}$ MS; Fabrício Boscolo Del Vecchio ${ }^{1}$ PhD

Recebido em: 16 de novembro de 2016. Aceito em: 12 de abril de 2017.

Publicado online em: 29 de agosto de 2017.

\section{Resumo}

Introdução: O Campo Operacional de Instrução (COI) demanda elevado esforço físico e mental.

Objetivo: Avaliar os efeitos das atividades de treinamento militar sobre indicadores de dano muscular periférico, de controle autonômico e de aptidão física em alunos do Núcleo de Preparação de Oficiais da Reserva (NPOR) de Pelotas.

Métodos: Este estudo longitudinal, avaliou 18 alunos do curso de formação do NPOR, antes e após uma semana de atividade em COI com exercícios físicos intensos. Mensuraram-se a concentração sanguínea de creatina quinase [CK], os indicadores da variabilidade da frequência cardíaca (VFC) e o desempenho dos participantes em testes de aptidão física. 0 teste de Shapiro-Wilk foi utilizado para avaliar a normalidade dos dados e os momentos foram comparados com teste $t$ de Student para amostras dependentes e as correlações testadas com a prova de Pearson. 0 nível de confiança das análises foi de $95 \%$.

Resultados: A potência de membros superiores e inferiores

Pontos-Chave Destaque

- Houve diminuição em potência de membros superiores e inferiores.

- Houve aumento em resistência abdominal e força isométrica de preensão da mão esquerda.

- Foi encontrada alta correlação entre a variação de potência de membros inferiores no salto contramovimento e [CK]. diminuiu $(\mathrm{p}<0,05)$ e o desempenho em força isométrica máxima de preensão da mão esquerda $(\mathrm{p}=0,03)$ e resistência abdominal $(p<0,00)$ aumentaram. Houve aumento na VFC $(p<0,01)$, e tamanho do efeito negativo em todos os casos. Entre as variáveis do domínio do tempo, LF teve tamanho do efeito igual a $-0,17$ e $\mathrm{HF}-0,28$ $(\mathrm{p}<0,001)$. Em $[\mathrm{CK}]$ não houve alteração. Houve alta correlação entre decréscimo em potência de membros inferiores (salto vertical com contramovimento) e $[\mathrm{CK}](\mathrm{p}=0,01)$.

Conclusão: Os resultados em VFC sugerem que as atividades realizadas durante o COI promoveram respostas dos ramos simpático e parassimpático de forma significativa, e indicaram boa saúde cardíaca dos participantes devido ao aumento observado entre intervalos RR. Além disso, a diminuição de potência de membros inferiores (no salto vertical com contramovimento) e níveis de [CK] indicou que diferenças nos valores do desempenho, no salto vertical com contramovimento, pode ser, também, utilizado como marcador não invasivo de dano muscular.

Palavras-chave: treinamento físico, militares, saúde, desempenho, fisiologia do exercício.

\footnotetext{
${ }^{\S}$ Autor correspondente: Léo Dutra Cabistany - e-mail: leocabistany@gmail.com.

Afiliações: ${ }^{1}$ Escola Superior de Educação Física da Universidade Federal de Pelotas; ${ }^{2}$ Faculdade Anhanguera Pelotas.
} 


\begin{abstract}
Introduction: The Operational Field of Instruction (OFI) demands high physical and mental effort.

Objective: To evaluate the effects of military training activities on indicators of peripheral muscle damage, autonomic control, and physical fitness in students of the Center for Reserve Officers Preparation (CROP) of Pelotas, before and after an OFI and to examine associations with muscle damage. on trainee students.

Methods: This longitudinal study evaluated 18 students of the NPOR training course, before and after one week of activity in IOC with intense physical exercises. The blood concentration of creatine kinase [CK], the indicators of heart rate variability (HRV) and the performance of participants in physical fitness tests were measured. The Shapiro-Wilk test was used to evaluate the normality of the data and the moments were compared with Student's t test for dependent samples and the correlations tested with the Pearson test. The confidence level of the analyzes was $95 \%$.

Results: The power of the upper and lower limbs decreased ( $p$

Keypoints

- Upper and lower limbs power

decreased.

- Abdominal resistance and

isometric strength of the left

hand increasead.

- Theres was high correlation of variation of lower limb power in countermovement jump and

$[C K]$. $<0.05)$ and the performance in maximal isometric strength of the left hand $(p=0.03)$ and abdominal resistance $(p$ $<0.001)$ increased. There was an increase in HRV $(\mathrm{p}<0.01)$, and size of the negative effect in all cases. Among the variables of the time domain, LF had effect size equal to -0.17 and HF $-0.28(\mathrm{p}<0.001)$. In [CK] there was no change. There was a high correlation between decrease in power of lower limbs (vertical jump with countermovement) and $[\mathrm{CK}](\mathrm{p}=0.01)$.

Conclusion: The HRV results suggest that the activities performed during the IOC promoted sympathetic and parasympathetic responses in a significant way and indicated good cardiac health of the participants due to the observed increase between RR intervals. In addition, lower limb power (in vertical jump with countermovement) and [CK] levels indicated that differences in vertical jump performance values with countermovement may also be used as a non-invasive marker of muscle damage. The abstract should not exceed 250 words and must summarize the work, giving a clear indication of the conclusions contained therein. It should be structured. The introduction should contextualize the problem.
\end{abstract}

\title{
Efeitos de campo operacional de instrução sobre aptidão física, dano muscular e controle autonômico de alunos do NPOR do Exército Brasileiro
}

\section{Introdução}

As rotinas de treinamento físico militar incluem diversos exercícios calistênicos, como flexões de cotovelos, puxadas na barra fixa, além de corridas, e outras atividades que, somadas, tem por intuito desenvolver aptidão física elevada(1). Um segmento específico do Exército Brasileiro é o Núcleo de Preparação de Oficiais da Reserva (NPOR), que tem por objetivo treinar jovens para contribuírem com o desenvolvimento militar em suas as áreas de competência(2). O Exército Brasileiro tem a finalidade de proteger e assistir ao país e sua população(2), e para o desempenho das atribuições dos militares, um bom nível de aptidão física é um dos requisitos fundamentais(3). Nesse contexto, o conhecimento do impacto do treinamento físico militar (TFM) no organismo é relevante para seu planejamento(4). Por outro lado, são escassas as informações sobre este modelo de treinamento e suas implicações sobre as variáveis fisiológicas que indicam desgaste orgânico(1,5).

Para avaliação de condicionamento e saúde dos militares, parâmetros como a variabilidade da frequência cardíaca (VFC) e a concentração sanguínea da enzima creatina quinase [CK] apresentam-se como indicativos quanto à aptidão física e estado de saúde cardiovascular 
dos indivíduos(6). A VFC Por ser indicador autonômico, explicita a relação entre sistema nervoso e demais estruturas orgânicas, podendo ser um indicador de fadiga do sistema nervoso central(7).

Do ponto de vista periférico - muscular -, o indicador mais comumente relacionado ao dano muscular é a enzina creatina quinase(8). Ela é uma enzima catalisadora, presente em diversos tecidos orgânicos inclusive no tecido muscular esquelético, sendo afetada e proporcional ao volume de massa muscular do indivíduo. Além disso, a [CK] serve como indicador de dano muscular, visto ter sitio ativo intramuscular, lesões decorrentes de atividades e/ou exercícios físicos geram extravasamento desta enzima para corrente sanguínea, tornando possível sua detecção, mesmo em amostras pouco volumosas(9). Nesse contexto, considerar a associação entre estas duas variáveis torna-se relevante, pois, suas respostas podem se dar em tempos semelhantes.

Além das avaliações fisiológicas, existem outras avaliações, que por meio de desempenho, representam os níveis de aptidão física do indivíduo. Saltos e exercícios calistênicos resistidos, são empregados para avaliar estado físico de militares(1,5,10). Além da VFC e da [CK], estas variáveis refletem a condição muscular e ajudam na compreensão do estado físico, além de fornecerem informações sobre as cargas de trabalho impostas pelo TFM (1). Resultados de estudo prévio conduzido em alunos do NPOR (1) demonstrou que esses indivíduos apresentaram aptidão física intermediária e que os níveis de [CK] de repouso estavam próximos ao limite superior, provavelmente em função das rotinas de treinamento e os valores da VFC se mostraram dentro da normalidade. Alunos do NPOR quando submetidos a 13 semanas de TFM apresentaram melhora no condicionamento físico(5). No entanto, pouco se sabe como as atividades de um Campo Operacional de Instrução (COI) impactam o organismo de militares. O COI é composto por tarefas nas quais os alunos do curso de formação do NPOR têm oportunidade de planejar e executar diversas missões, em contexto de operações ofensivas e defensivas, conhecidas por proporcionarem desgastes físicos e mentais elevados. Dessa forma, o estudo objetivou avaliar os efeitos das atividades de treinamento militar sobre indicadores de dano muscular periférico, de

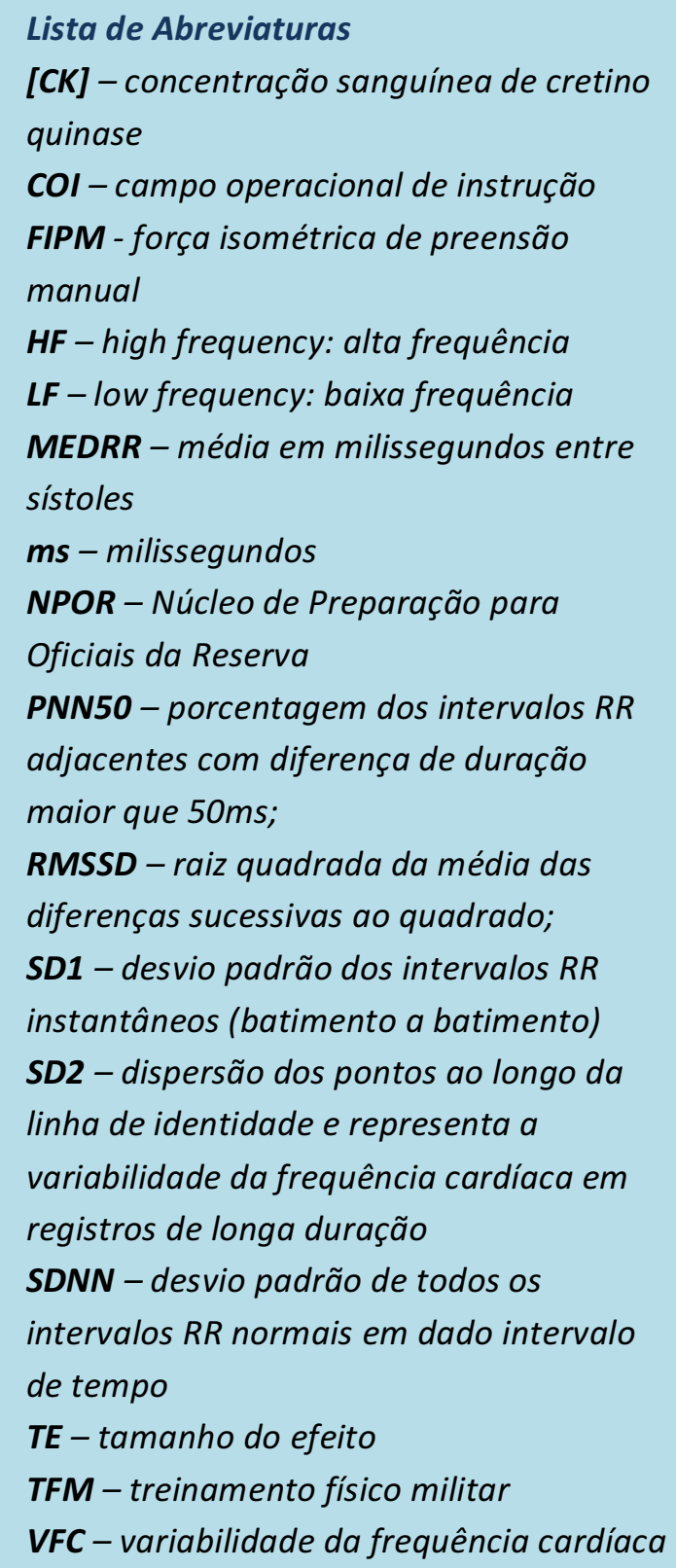

controle autonômico e de aptidão física em alunos do Núcleo de Preparação de Oficiais da Reserva (NPOR) de Pelotas.

\section{Métodos}

\section{Desenho de estudo e amostra}

Estudo observacional, longitudinal, para o qual foram convidados a participar todos os alunos do curso de formação do NPOR de Pelotas/RS, no ano de 2015 (N=18). 
O critério de inclusão foi ser aluno do curso de formação e o critério de exclusão foi estar dispensado das rotinas do treinamento físico militar (TFM) diário.

\section{Aspectos éticos}

Após explanação sobre os objetivos, procedimentos, riscos e benefícios do estudo, todos concordaram em participar e assinaram o termo de consentimento livre e esclarecido. Todos os preceitos que regem a pesquisa envolvendo seres humanos foram seguidos. $\mathrm{O}$ projeto foi aprovado pelo Comitê de Ética da Escola de Educação Física da Universidade Federal de Pelotas, parecer 004/2012.

\section{Variáveis desfecho}

\section{Controle autonômico}

O controle autonômico é avaliado por meio da VFC, que é analisada em três domínios: i) domínio do tempo, que mede a partir de equações geométricas e índices estatísticos na unidade de tempo de milissegundos o intervalo RR entre batimentos cardíacos sucessivos este intervalo acontece entre duas ondas $\mathrm{R}$ de batimentos sucessivos e corresponde à frequência de despolarização ventricular; ii) domínio da frequência, medido em hertz, podendo ser classificada como de alta (HF - do inglês: high frequency), baixa (LF - do inglês: low frequency) ou ainda muito baixa (VLF do inglês, very low frequency) frequência; e iii) domínio não-linear. Por ser indicador autonômico, a VFC explicita a relação entre sistema nervoso e demais estruturas orgânicas, podendo ser utilizado como um indicador de fadiga do sistema nervoso central(7).

\section{Dano muscular}

A [CK] é um importante indicador de dano muscular periférico, e tem seu pico de extravasamento na corrente sanguínea aproximadamente 24 horas após a execução do exercício/esforço. Os níveis de [CK] foram avaliados nos momentos antes e depois do COI.

Indicadores de aptidão física

Os indicadores de aptidão física avaliados foram: força e potência de membros superiores $\mathrm{e}$ inferiores e resistência abdominal. A avaliação destes indicadores está descrita em detalhes na seção procedimentos de coleta de dados.

\section{Variável de exposição}

A variável de exposição foi o COI, rotina de atividades operacionais, composta de diversos tipos de exercícios físicos extenuantes e de tarefas cognitivas, desenvolvidas ao longo de seis dias.

\section{Procedimentos de coleta de dados}

Realizaram-se coletas das variáveis fisiológicas, antropométricas (massa corporal e estatura) e de aptidão física em dois momentos: imediatamente antes do início da rotina composta por operações militares de campo, componentes do $\mathrm{COI}$ e, 24 horas depois de retornarem do COI. O COI teve duração de 6 dias e envolveu atividades extenuantes, como caminhada de longas distâncias com equipamento militar, pesando aproximadamente 10 quilos, restrições de sono e de alimento, além de atividades de simulação de ataque e defesa com armas brancas e de fogo. Dois avaliadores, previamente treinados, foram responsáveis pelas rotinas repetidas nos dois momentos, e supervisionados de modo contínuo e proximal por pesquisador orientador.

Inicialmente, aconteceu coleta dos dados referentes à VFC, para avaliar o controle autonômico. Esta avaliação foi realizada utilizando-se o monitor cardíaco (Polar ${ }^{\circledR}$, modelo RS800CX)(12). Para isto, os sujeitos permaneceram em silêncio e sentados por cinco minutos, período suficiente para verificação dos parâmetros da VFC(7). Após, os dados foram coletados com o monitor e transferidos por dispositivo infravermelho para computador pessoal. Inicialmente, os dados foram analisados por meio de inspeção visual no programa computacional Polar Pro-Trainer 5.0 e, quando necessário, aplicou-se filtro de correção de erros, com potência moderada e zona mínima de proteção de $6 \mathrm{bpm}$. Subsequentemente, exportou-se arquivo do tipo .txt, com todos os dados da VFC, o qual foi aberto no programa Kubios(13), no qual os dados foram analisados. Consideraram-se as frequências relativas ao domínio do tempo: SDNN, desvio padrão de todos os intervalos RR normais em dado intervalo de tempo; RMSSD, raiz quadrada da média das diferenças sucessivas ao quadrado; PNN50, porcentagem dos intervalos $\mathrm{RR}$ adjacentes 
com diferença de duração maior que 50milissegundos; MEDRR, média em milissegundos entre sístoles. No domínio da frequência cardíaca foram analisadas: HF e LF e a relação HF/LF. Para o domínio não-linear, empregaram-se SD1 (desvio padrão dos intervalos RR instantâneos) e SD2 (desvio padrão dos intervalos RR em longo prazo)(14).

De modo subsequente à coleta da VFC, ocorreu determinação da atividade enzimática em [CK], a partir da análise de amostras sanguíneas de $50 \mu \mathrm{L}$, as quais foram coletadas do lobo da orelha com capilares heparinizados (Registro ANVISA 10287410157, Reflotron $\left.{ }^{\circledR}\right)$, empregando punção com lancetas estéreis descartáveis (Accu-Chek Softclix). Imediatamente após a coleta, o sangue foi transferido para tiras próprias (Registro ANVISA 10287410157, Reflotron $($ ) e analisado por procedimento de ensaio colorimétrico (Reflotron Analyser ${ }^{\circledR}$, Boehringer-Mannheim, França).

A seguir, aplicaram-se os testes de aptidão física, na ordem descrita que se segue: A força de membros superiores foi avaliada pelo teste de força isométrica de preensão manual (FIPM, em kgf) e pelos testes de flexão e extensão de braços ao solo e na barra fixa, em dois momentos distintos. A FIPM foi mensurada com dinamômetro JAMAR ${ }^{\mathrm{TM}}$ (Sammons Preston, EUA), padrão ouro para esta mensuração, sendo que, para este teste, o indivíduo ficava sentado com cotovelo fletido em 90 graus e deveria aplicar força máxima em três momentos consecutivos, com $60 \mathrm{~s}$ de intervalo entre eles(15). Coletaram-se os dados dos hemicorpos direito e esquerdo, nesta ordem, e o valor médio de cada um foi considerado nas análises. O método apresenta correlação intraclasse de 0,93 e alta reprodutibilidade(16).

Em seguida, avaliou-se a potência de membros inferiores a partir do salto com contramovimento $(\mathrm{CMJ}$, em $\mathrm{cm})$, realizado sobre tapete de contato (Jump System, CEFISE ${ }^{\circledR}$, Nova Odessa) que registra o tempo de voo e mensura altura do salto que de acordo com estudo prévio, apresenta valor de $r$ entre 0,83 e $0,99(17)$; além de salto horizontal, que consistiu em mensurar a distância do salto com fita métrica inelástica. Para isto, os avaliados se posicionaram atrás de marcação feita no solo e, após comando verbal, o indivíduo saltava e mediu-se. Registrou-se a distância entre a linha de partida e a marca mais próxima desta deixada pelo avaliado. Foram realizadas três tentativas para cada tipo de salto, e a média do desempenho foi considerada nas análises.

Logo após, avaliou-se a força de membros inferiores pelo teste de meio agachamento, que consiste em realizar o número máximo de repetições em 2 minutos. A execução exigia angulação de membros inferiores de até $90^{\circ}$ enquanto toda planta do pé deveria permanecer em contato com o solo.

Por fim, a resistência abdominal foi avaliada com os participantes em decúbito dorsal, com joelhos flexionados e pés apoiados no solo, com as mãos próximas ao pescoço deveriam elevar o tronco até encostar os cotovelos nos joelhos.

\section{Análise estatística}

Os efeitos do COI foram analisados quanto às suas alterações no pré e pós-atividade e, também, foram feitas análises quanto à associação. Após teste de normalidade de Shapiro-Wilk, foi realizado teste $t$ de Student pareado para comparar os valores pré e pós COI. Além disso, foi calculada o coeficiente de variação $(\Delta \%)$ entre os momentos (pós-pré). Para cálculo de tamanho de efeito (TE) foi aplicada equação: $\mathrm{TE}=$ (Média pré - Média pós) / DP pós), e interpretado do seguinte modo: $0,2=$ pequeno, $0,5=$ moderado e $0,8=$ grande(18). Para análises de correlação entre os coeficientes de variação das variáveis em pré e pós COI, utilizou-se coeficiente de Pearson. O nível de confiança das análises foi de $95 \%$. Empregou-se o programa computacional SPSS 17.0 para análise dos dados.

\section{Resultados}

Os resultados referentes ao desempenho em aptidão física no pré e pós COI apresentam-se na Tabela 1. Observa-se que a FIMP da mão direita, o teste de $2 \mathrm{~min}$ de meio agachamentos e concentração de [CK] não mostraram alteração entre momentos $(p>0,05)$. Os valores pós-campo da força isométrica máxima de preensão da mão esquerda $(\mathrm{p}=0,03)$, e de resistência abdominal $(p<0,001)$ foram melhores que os da situação pré-campo. Força 
de membros inferiores (salto horizontal, $\mathrm{p}=0,00$ ) e de membros superiores (repetições na barra fixa, $p=0,00)$, salto com contramovimento $(\mathrm{p}=0,01)$ e número máximo de flexão de cotovelos $(\mathrm{p}=0,00)$ exibiram média geral diminuída.

Quanto à VFC, os dados são apresentados na Tabela 2. Com exceção da LF/HF, que aumentou mais de $350 \%$, todas as variáveis apresentaram diminuições estatisticamente significativas. Todos os parâmetros da VFC mostraram TE de pequena magnitude. No domínio da frequência o tamanho do efeito foi negativo em todos os parâmetros $(p<0,01)$, a saber: $\mathrm{SDNN}=-0,17 ; \mathrm{RMSSD}=-0,21$; PNN50 = -0,23; SD1 $=-0,21 ;$ SD2 $=-0,16$ e $\mathrm{MEDRR}=-0,09$. Entre as variáveis do domínio do tempo, LF teve tamanho do efeito igual a 0,17 e HF $-0,28 \quad(p<0,01)$. Não houve correlação significativa entre as variáveis da $\mathrm{VFC}$ e $[\mathrm{CK}]$.

O desempenho no teste de potência de membros inferiores (salto vertical com contramovimento) mostrou redução no momento pós COI $(p=0,01)$. Houve forte correlação do delta de variação deste indicador com o delta percentual de [CK].

\section{Discussão}

O presente estudo investigou o impacto de seis dias no COI sobre a VFC, sobre indicadores de aptidão física e marcador de dano muscular [CK] em militares do NPOR. O principal achado do presente estudo foi a redução da potência de membros inferiores, inferida pelos saltos vertical com contramovimento e em distância, bem como diminuição de força e de potência de membros superiores. Destaca-se, ainda, diminuição estatisticamente significativa das variáveis representantes da variabilidade da frequência cardíaca, o que se associa com impacto elevado do COI no controle autonômico. A correlação entre a redução nos valores do salto vertical com contramovimento e alta $[\mathrm{CK}]$, indica que houve dano muscular nos militares participantes do COI. O extravasamento de [CK] se dá quando há ruptura de fibras musculares e do sarcolema, e que este tipo de lesão ocorre por estresse causado por ações musculares que podem também promover fadiga e ruptura de fibras periféricas(19).
Assim, as alterações nos níveis sanguíneos de [CK] podem ser explicadas pelo desgaste periférico, dano muscular, pois menor quantidade de fibras musculares seriam recrutadas, em função de ruptura e fadiga, diminuindo a potência de movimento no salto em questão (20).

A maioria dos indicadores de desempenho físico sofreram decréscimo que pode ser explicado por serem atividades que exigem recrutamento de grandes grupamentos musculares. Foi evidenciada redução do desempenho em indicadores de resistência de membros superiores e potência de membros superiores e inferiores, o que pode ser explicado por prejuízos na ativação muscular decorrente de fadiga resultante das atividades realizadas no COI, este que exigiu recrutamento de unidades motoras por longo período de tempo, causando fadiga muscular acentuada, pois, unidades contráteis recrutadas desde o início da atividade provavelmente apresentaram menor poder contrátil(21), além de fadiga mental e emocional.

Os participantes do estudo, apresentaram uma média de repetições no teste de flexão e extensão de braço ao solo $(48,7)$ bem maior do que o encontrado por Ávila et al.(5) $(33,6)$. Assim, ao considerarmos a força de membros superiores, o número de repetições foi bastante superior. Os alunos do NPOR do presente estudo apresentaram desempenho no teste de força de preensão manual um pouco menor (FMPM direita $46,62 \mathrm{~kg}$ e esquerda 45,30) do que o grupo de militares integrantes do Curso de Monitores da Escola de Educação Física do Exército, lado direito com $52,9 \pm 7,1 \mathrm{Kgf}$ e esquerdo com $51,0 \pm 6,5 \mathrm{Kgf}(10)$, e os valores aumentaram do momento pré COI para o pósCOI. Provavelmente, o aumento na força isométrica de preensão manual pode ser decorrente do baixo requerimento desse grupamento muscular específico durante os exercícios de campo, por envolver basicamente caminhadas com equipamentos, não fadigando os músculos envolvidos nesta tarefa. Por se tratar de teste que requer ativação de músculos pequenos, há menor impacto do desgaste de SNC não prejudicando atividade contrátil destes grupos musculares(21).

$\mathrm{O}$ teste de resistência abdominal mostrou diferença estatística entre momentos, com 
aumento do número de repetições no momento pós-teste. Entendendo que a rotina exigiu mais resistência aeróbia, por se tratar de atividades de longa duração, o que não geraria fadiga nesta musculatura específica, e possivelmente, atuaria no sentido de melhorar do desempenho(22). Uma possível causa para esse aumento de desempenho pode, possivelmente, ser creditada à melhora na coordenação intramuscular e intermuscular. $\mathrm{O}$ incremento do desempenho em alguns testes neuromusculares pode ter ocorrido por aumento do nível de estimulação neural e, consequentemente, maior número de unidades motoras recrutadas, pois, uma maior quantidade de fibras musculares ativadas num mesmo período de tempo, eleva a capacidade de desempenho(23).

É plausível que os indivíduos, após campo operacional, apresentem modificações em seu funcionamento mental, tornando-se fortalecidos, pois, há indícios de que fatores psicológicos influenciam o desempenho físico de indivíduos quando estes são testados e sentem-se capazes para realização de determinadas atividades(24).

O estudo da [CK], como indicador de dano muscular periférico, pode ajudar a compreender melhor em que medida a carga de exercício é suficiente para ocasionar ajustes no perfil muscular (25). Valores de [CK] entre 300 e 500 U/L são tidas como aceitáveis em situação pós esforço (26), e a média em ambos os momentos avaliativos permaneceu dentro dessa margem. Além disso, a elevação dos níveis de $[\mathrm{CK}]$ foi pequena $(\Delta \sim 35 \%)$, pontuando a ideia de que as atividades, embora extenuantes, não ocasionaram dano muscular(26).

A correlação apresentada na Figura 1 indica que com maior [CK] menor foi o desempenho dos alunos no salto, devido a maior lesão muscular, diminuindo a capacidade de trabalho que demande geração de potência devido à queda na produção de velocidade e de força máxima(27). Um estudo em jogadores de futsal - esporte em que se realiza padrão de movimento baseado em ciclo encurtamentoalongamento, após quatro dias consecutivos de jogos, houve queda de performance(28). De forma análoga, acredita-se que o COI, por envolver períodos de longas caminhadas, pode ter influência sobre o salto com contramovimento, evidenciando a fadiga muscular e provável dano muscular.

Quanto à VFC, Kaikkonen et al.(29), ao submeterem indivíduos $(\mathrm{N}=16)$ treinados em exercício de resistência a diferentes protocolos de treinamento: intensidade alta: $3 \mathrm{~km}$ de corrida a $85 \%$ do volume máximo de consumo de oxigênio $\left(\mathrm{VO}_{2 \mathrm{max}}\right)$; moderada: $3 \mathrm{~km}$ a $60 \%$ do $\mathrm{VO}_{2 \mathrm{max}}$; e esforço prolongado: $14 \mathrm{~km}$ a $60 \%$ da $\mathrm{VO}_{2 \max }$, encontraram menores da VFC após a realização dos $14 \mathrm{~km}$ em comparação com a corrida de $3 \mathrm{~km}$. Da mesma forma, no presente estudo, os valores de todas as variáveis da VFC tiveram redução estatisticamente significativas após as atividades do COI. Havendo diferenças na VFC, há aumento em sua variabilidade que indica maior nível de treinamento(7) e estão relacionadas com melhora no desempenho central e periférico em atletas de endurance. Nesse sentido, os resultados sugerem que os alunos obtiveram melhoras no condicionamento entre os momentos de avaliação(30). Dentre as variáveis da VFC, HF é o indicador da atuação do nervo vago sobre a bomba cardíaca e LF sofre influência da ação conjunta dos ramos nervosos simpático e parassimpático; os quais apresentaram aumento estatisticamente significativo $(p=0,007$ e $p=0,004$, respectivamente). Aumentos na variabilidade indicam comportamento saudável do coração e boa adaptação a estímulo, inclusive de exercícios físicos(31). Os participantes do presente estudo apresentaram diminuição da atividade em ambas frequências (HF e LF) no segundo momento de registro, sendo que em $\mathrm{HF}$ a diminuição foi mais acentuada, exibindo diminuição da atividade simpática e indicando saúde cardíaca(7).

A razão HF/LF é utilizada para cálculo do balanço simpatovagal e, assim como em grupo de jovens adultos submetidos a dois diferentes protocolos de exercícios resistidos(32), no presente estudo houve aumento em TE (tamanho do efeito), indicador de maior atividade parassimpática, (responsável pela desaceleração e estabilidade elétrica cardíaca(7). Tais resultados estão alinhados com estudo experimental prévio, no qual 
Tabela 1 - Desempenho em aptidão física pré e pós-COI e correlação com indicador de dano muscular periférico[CK]

\begin{tabular}{|c|c|c|c|c|c|c|c|c|c|}
\hline \multirow{2}{*}{ FIPM } & \multicolumn{2}{|c|}{$\frac{\text { PRE-COI }}{\text { Média } \pm \text { DP }}$} & \multicolumn{2}{|c|}{$\frac{\text { PÓS-COI }}{\text { Média } \pm \text { DP }}$} & Teste $t P$ & $\mathrm{TE}$ & $\Delta(\%)$ & \multicolumn{2}{|c|}{ Correlação com [CK] } \\
\hline & & & & & & & & & \\
\hline Direita (kgf) & 44,78 & $\pm 7,46$ & 46,62 & $\pm 8,12$ & 0,15 & 0,25 & 4,93 & 0,04 & 0,86 \\
\hline Esquerda (kgf) & 42,70 & $\pm 7,26$ & 45,30 & $\pm 8,20$ & $\mathbf{0 , 0 3}$ & 0,36 & 6,71 & $-0,21$ & 0,38 \\
\hline Salto $\mathrm{c} /$ contramovimento $(\mathrm{cm})$ & 29,57 & $\pm 3,19$ & 27,85 & $\pm 2,88$ & $\mathbf{0 , 0 1}$ & 0,53 & $-5,33$ & $-0,54$ & $\mathbf{0 , 0 1}$ \\
\hline Salto horizontal $(\mathrm{cm})$ & 197,39 & $\pm 11,98$ & 172,75 & $\pm 18,98$ & $\mathbf{0 , 0 0}$ & 2,05 & $-12,39$ & $-0,03$ & 0,88 \\
\hline Força de membros inferiores & & & & & & & & & \\
\hline 2-min Flexões de Cotovelos (reps) & 50,11 & $\pm 13,00$ & 46,83 & $\pm 14,24$ & $\mathbf{0 , 0 0}$ & 0,25 & $-5,13$ & 0,06 & 0,79 \\
\hline Força de membros superiores & & & & & & & & & \\
\hline $\begin{array}{l}\mathrm{N}^{\circ} \text { Máximo de Barras (reps) } \\
\text { Resistência abdominal }\end{array}$ & 10,72 & $\pm 3,04$ & 8,47 & $\pm 2,96$ & $\mathbf{0 , 0 0}$ & 0,73 & $-24,31$ & 0,18 & 0,47 \\
\hline $\begin{array}{l}\text { 2-min de abdominais (reps) } \\
\text { Indicador de dano muscular periférico }\end{array}$ & 48,89 & $\pm 9,85$ & 100,06 & $\pm 30,75$ & $\mathbf{0 , 0 0}$ & 5,20 & 112,79 & 0,13 & 0,60 \\
\hline$[\mathrm{CK}](\mathrm{U} / \mathrm{L})$ & 415,17 & $\pm 403,62$ & 439,50 & $\pm 207,42$ & 0,77 & 0,06 & 35,09 & - & - \\
\hline
\end{tabular}


Tabela 2 - Variáveis referentes aos domínios do tempo e da frequência da variabilidade da frequência cardíaca $(\mathrm{n}=18)$

\begin{tabular}{|c|c|c|c|c|c|c|c|c|c|}
\hline & \multicolumn{2}{|c|}{ PRÉ-COI } & \multicolumn{2}{|c|}{ PÓS-COI } & \multirow[b]{2}{*}{$\mathrm{TE}$} & \multirow[b]{2}{*}{$\Delta(\%)$} & \multirow[b]{2}{*}{ Teste $t P$} & \multicolumn{2}{|c|}{ Correlação com $[\mathrm{CK}]$} \\
\hline & Média & $\pm \mathrm{DP}$ & Média & $\pm \mathrm{DP}$ & & & & $r^{*}$ & $\mathrm{P}$ \\
\hline MEDRR (ms) & 1858,14 & $\pm 4350,82$ & 1432,53 & $\pm 3409,17$ & $-0,09$ & 200,17 & $<0,001$ & $-0,14$ & 0,56 \\
\hline SDNN (ms) & 204,32 & $\pm 479,10$ & 123,00 & $\pm 290,61$ & $-0,17$ & 41,52 & $<0,001$ & $-0,11$ & 0,65 \\
\hline RMSSD (ms) & 164,31 & $\pm 386,10$ & 82,81 & $\pm 194,79$ & $-0,21$ & 42,49 & $<0,001$ & 0,00 & 0,99 \\
\hline PNN50 (unidades) & 84,97 & $\pm 199,46$ & 37,52 & $\pm 88,37$ & $-0,23$ & 24,97 & $<0,001$ & $-0,14$ & 0,56 \\
\hline $\mathrm{LF}\left(\mathrm{ms}^{2}\right)$ & 6903,68 & $\pm 16245,77$ & 4026,46 & $\pm 9632,46$ & $-0,17$ & 1476,17 & 0,007 & 0,00 & 0,99 \\
\hline $\mathrm{HF}\left(\mathrm{ms}^{2}\right)$ & 5943,82 & $\pm 14259,23$ & 1843,44 & $\pm 4359,58$ & $-0,28$ & 2188,39 & 0,004 & 0,13 & 0,60 \\
\hline LF/HF razão & 1,74 & $\pm 1,52$ & 3,64 & $\pm 3,01$ & 1,25 & 351,59 & 0,040 & 0,04 & 0,87 \\
\hline SD1 (ms) & 116,42 & $\pm 273,560$ & 58,67 & $\pm 138,00$ & $-0,21$ & 30,10 & $<0,001$ & $-0,002$ & 0,99 \\
\hline $\mathrm{SD} 2(\mathrm{~ms})$ & 263,02 & $\pm 616,641$ & 163,06 & $\pm 451,16$ & -0.16 & 50,78 & $<0,001$ & $-0,13$ & 0,59 \\
\hline
\end{tabular}

ms - milissegundos. * Coeficiente de correlação entre variáveis selecionadas e [CK]. $\Delta(\%)$ : coeficiente de variação. LF: baixa frequência; HF: alta frequência; TE: tamanho do efeito; MEDRR: média em milissegundos entre sístoles; SDNN: desvio padrão de todos os intervalos RR normais em dado intervalo de tempo; RMSSD: raiz quadrada da média das diferenças sucessivas ao quadrado; PNN50: Representa a porcentagem dos intervalos RR adjacentes com diferença de duração maior que 50ms; SD1:representa a dispersão dos pontos perpendiculares à linha de identidade batimento a batimento; SD2: dispersão dos pontos ao longo da linha de identidade e representa a VFC em registros de longa duração. 


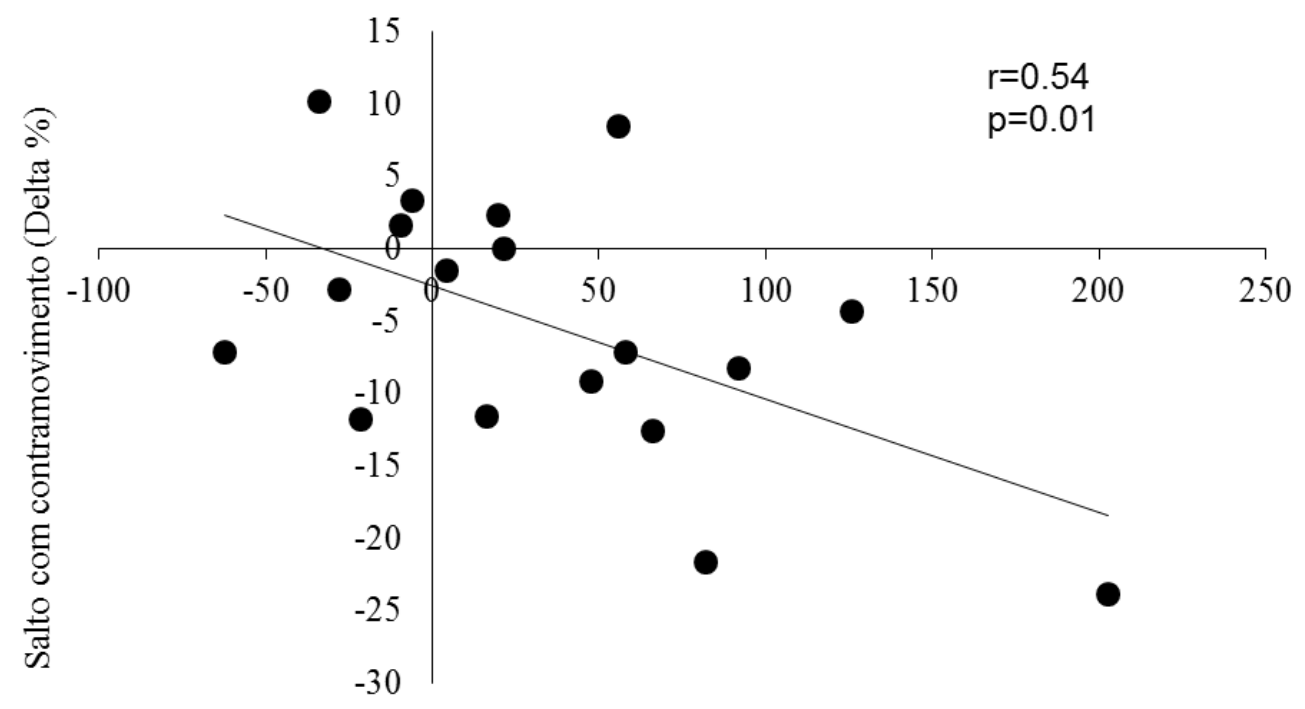

Concentração de creatina quinase (Delta \%)

Figura 1 - Correlação entre delta \% do salto com contramovimento e da [CK]

resultados semelhantes foram encontrados quanto aos efeitos do treino resistido sobre a VFC, sugerindo melhora na função parassimpática(31).

\section{Pontos fortes e limitações do estudo}

Os pontos fortes dizem respeito a compreensão do estresse envolvido nas rotinas de COI a que os indivíduos estão sujeitos durante o período de formação.

Como limitações estão que os testes eram de campo e as inferências são possíveis apenas para a parcela da população de militares que apresentem perfil semelhante ao desta amostra.

\section{Conclusão}

Os resultados apontaram que o COI gerou desgaste físico e implicou em queda de desempenho em potência de membros inferiores e superiores. Força isométrica máxima de preensão da mão esquerda e resistência abdominal apresentaram valores superiores após a atividade, embora as causas disto precisem ser investigadas, acredita-se que os indivíduos tenham ficado mais resilientes. Além disso, encontrou-se correlação importante entre $\Delta \%$ do salto com contramovimento e $[\mathrm{CK}]$, indicando que esta variável de desempenho físico pode também ser utilizada como marcador para dano muscular. Sugere-se que outros estudos confirmem esta hipótese.

\section{Agradecimentos}

Agradecemos ao Exército Brasileiro, em especial ao $9^{\circ}$ Batalhão de Infantaria Motorizada de Pelotas/RS, por gentilmente nos terem acolhido e possibilitado a pesquisa.

\section{Declaração de conflito de interesses}

Não há nenhum conflito de interesses no presente estudo.

\section{Declaração de financiamento \\ Pesquisa não financiada.}

\section{Referências}

1. Del Vecchio FB, Oliveira LF, Ferreira H, Barth J, Coswig VS. Relações entre aptidão física, concentração de creatina quinase e variabilidade da frequência cardíaca em alunos do NPOR de Pelotas/RS. Pensar a Prática. 2015; 18(2):278-293. https://doi.org/10.5216/rpp.v18i2.34147.

2. Brasil. Defesa e segurança: em tempos de paz, exército defende fronteiras nacionais. Disponível em:

(http://www.brasil.gov.br/defesa-eseguranca/2012/04/em-tempos-de-pazexercito-defende-fronteiras-nacionais. Acessado 11/08/215). 
3. Lindquist $\mathrm{CH}$, Bray RM. Trends in overweight and physical activity among U.S. military personnel, 1995-1998. Preventive Medicine.2001;.1(32):57-65. doi: 10.1006/pmed.2000.0771.

4. Roschel H, Tricoli V, Ugrinowitsch C. Treinamento físico: considerações práticas e científicas. Revista Brasileira de Educação Física. 2011; 25(spe): 53-65. http://dx.doi.org/10.1590/S1807-

55092011000500007.

5. Avila JA, Lima Filho PDB, Páscoa MA, Tessutti LS. Efeito de 13 semanas de treinamento físico militar sobre a composição corporal e o desempenho físico dos alunos da escola preparatória de cadetes do exército. Revista Braileira de Medicina do Esporte. 2013; http://dx.doi.org/10.1590/S151786922013000500013.

6. Guzmán JEO, Romero DM, Calderón CA, Urbina A Análisis de los componentes espectrales de la variabilidad cardíaca en hombres jóvenes entrenados: comparación del entrenamiento aeróbico y anaeróbico. Apunts Medicina Del'Esporte . 2012; 47(174):41 -47. doi:

https://doi.org/10.1016/j.apunts.2011.06.002

7. Vanderlei LCM, Pastre CM, Hoshi RA, Carvalho TD, Godoy MF. Noções básicas de variabilidade da frequência cardíaca e sua aplicabilidade clínica. Revista Brasileira de Cirurgia Cardiovascular. 2009; 24(2):205$217 . \quad \mathrm{http}: / / \mathrm{dx}$. doi.org/10.1590/S010276382009000200018

8. Coswig VS, Neves AHS, Del Vecchio FB. Respostas bioquímicas, hormonais e hematológicas a lutas de jiu-jitsu brasileiro. Revista Brasileira de Ciência e Movimento. 2013 21(2):19-30.doi: http://dx.doi.org/10.18511/01031716/rbcm.v21n2p19-30

9. Coelho DB, Morandi RF, de Melo MAA, Garcia ES. Cinética da creatina quinase em jogadores de futebol profisional em uma temporada competitiva. Revista Brasileira de Cineantropometria e Desempenho Humano. 2011; 13(3):189-194. http://dx.doi.org/10.5007/19800037.2011v13n3p189.
10. Martins MEA, Santos FM, Arantes RP. Relação da performance na barra fixa com a força de preensão manual e tempo de sustentação na barra fixa. Revista de Educação Física - Centro de Capacitação Física do Exército. 2004;128:65-72.

11. Redkva PE, Vargas LM, da Luz R, Gomes EB, Ikaminagakura EI. A influência da composição corporal no teste de cooper e testes motores realizados no NPOR DO $13^{\circ} \mathrm{BIB}$. Revista de Educação Física - Centro de Capacitação Física do Exército. 2010; 150:3440.

12. Barth J, Del Vecchio FB. Efeitos da frequência ventilatória sobre os índices da variabilidade da frequência cardíaca. Revista Ibero de Arritmia. 2014; 5(1):185-193. doi: 10.5031/v5i1.RIA10245.

13. Tarvainen MP, Niskanen JP, Lipponen JA, Ranta-aho PO, Karjalainen PA. Kubios HRV - A Software for Advanced Heart Rate Variability Analysis. 4th European Conference of the International Federation for Medical and Biological Engineering. 2009; 22 of the series IFMBE Proceedings: 1022-1025. doi: 10.1007/978-3-540-89208-3 243.

14. Oliveira RS, Pedro RE, Milanez VF, Bortolotti H, Costa MV, Nakamura FY Relação entre variabilidade da frequência cardíaca e aumento no desempenho físico em jogadores de futebol. Revista Brasileira de Cineantrometria e Desempenho Humano. 2012; 6(14):713-722. http://dx.doi.org/10.5007/1980$0037.2012 \mathrm{v} 14 \mathrm{n} 6 \mathrm{p} 713$.

15. Fernandes AA, Marins JCB. Teste de força de preensão manual: análise metodológica e dados normativos em atletas. Fisioterapia em Movimento. 2011; 24(3):567-578. http://dx.doi.org/10.1590/S010351502011000300021.

16. Zanchet MA, Del Vecchio FB. Efeito da Kinesio Taping sobre força máxima e resistência de força em padelistas. Fisioterapia em Movimento. 2013; 26(1):115-121. http://dx.doi.org/10.1590/S010351502013000100013. 
17. Castro-piñero J, Ortega FB, Artero EG, Girela-rejón MJ, Mora J, Sjöström M, Ruiz JR. Assessing muscular strength in youth: usefulness of standing long jump as a general index of muscular fitness. Journal of Strenght and Conditioning Research. 2010; 7(24):18101817. doi: 10.1519/JSC.0b013e3181ddb03d.

18. Rhea MR. Determining the Magnitude of Treatment Effects in Strength Training Research Through the Use of Effect Size. Journal of Strenght and Conditioning Research. 2004; 18(4):918-920. doi: 10.1519/14403.1.

19. Brancaccio P, Maffulli N, Limongelli FM. Creatine kinase monitoring in sport medicine. British Medical Bulletin. 2007; 81(82):209230. doi: 10.1093/bmb/ldm014.

20. Allen DG, Lamb GD, Westerblad H. (2008) Skeletal muscle fatigue: cellular mechanisms. Physiological Reviews 2008, 88(1):287-332.

doi: 10.1152/physrev.00015.2007.

21. Garland SJ, e Gossen ER. The muscular wisdom hypothesis in human muscle fatigue. Exercise and Sport Science Reviews. 2002; 30(1):45-49.

22. Nunes J, Rosa M, Del Vecchio FB. Treinamento de força com uso de correntes e potencialização pós-ativação do salto vertical. Revista Brasileira de Ciências do Esporte. 2012; 34(4):1017-1033. http://dx.doi.org/10.1590/S010132892012000400015.

23. Maior AS, Alves AA contribuição dos fatores neurais em fases iniciais do treinamento de força muscular: uma revisão bibliográfica. Motriz. 2003; 9(3):161-168.

24. McCormick A, Meijen C, Marcora, S. Psychological Determinants of Whole-Body Endurance Performance. Sports Medicine. 2015; 45(7):997-1015. doi: 10.1007/s40279015-0319-6.

25. Bartone PT, Roland RR, Picano JJ, Williams TJ. Psycological hardiness predicts success in US Army Special Forces Candidates. International Journal of Life Cycle Assessment. 2008; 1(16):78-81. doi: 10.1111/j.1468-2389.2008.00412.x.
26. Mougios M. Reference intervals for serum creatine kinase in athletes. Brazilian Journal of Sports Medicine. 2007;41(10):674-8. doi: 10.1136/bjsm.2006.034041.

27. Dal Pupo J, Detanico D, Santos SG. Parâmetros cinéticos determinantes do desempenho nos saltos verticais. Revista Brasileira de Cineantropometria e Desempenho Humano. 2012 14(1):41-51. doi: http://dx.doi.org/10.5007/1980-

0037.2012v14n1p41.

28. Freitas VH, Souza EA, Oliveira RS, Pereira LA, Nakamura FY et al. Efeito de quatro dias consecutivos de jogos sobre a potência muscular, estresse e recuperação percebida, em jogadores de futsal . Revista Brasileira de Educação Física e Esporte. 2014; 28(1):23-30. doi: http://dx.doi.org/10.1590/S180755092014005000002.

29. Kaikkonen P, Hynynen E, Mann T, Rusko $\mathrm{H}$, Nummela A. Can HRV be used to evaluate training load in constant load exercises? Europen Journal of Applied Physiology. 2010; 108:435-442. doi: 10.1007/s00421-009-12401.

30. Hedelin R, Bjerle P, Larse'n KH. Heart rate variability in athletes: relationship with central and peripheral performance. Medicine \& Science in Sports \& Exercise. 2001; 33(8):1394-1398.

31. Lopes PFF, Oliveira MIB, Sousa S.M, Nascimento DLA, Silva CSS, Rebouças GM, et al. Clinical Applications of Heart Rate Variability. Reviews in Neurociences. 2013; 21(4):600-603.

32. Maior AS, Netto C F, Eichwald A, Druck G, Villaça G, Foschiera R. S et al..Influência da Intensidade e do Volume do Treinamento Resistido no Comportamento Autonômico Cardíaco. Revista SOCERJ. 2009; 22(4):201209. 